\title{
Robust Image Hiding Method
}

\author{
Yu-An Ho ${ }^{1}$, Yung-Kuan Chan ${ }^{2}$, Chwei-Shyong Tsai ${ }^{2}$, Yen-Ping Chu ${ }^{1}$ \\ Institute of Computer Science, National Chung Hsing University, \\ No. 250, Kuokuang Rd., Taichung, Taiwan, R.O.C. ${ }^{1}$ \\ Department of Management Information Systems, National Chung Hsing University, \\ No. 250, Kuokuang Rd., Taichung, Taiwan, R.O.C. ${ }^{2}$
}

\begin{abstract}
Image hiding is hiding a secret image embedding to a cover image. This paper intends to propose an image hiding method. First, a secret image is compressed by BST. Meanwhile, dividing the compressed data into different ranks depends on the significance. Then, the cover image is transformed into frequency domain data by discrete wavelet transformation. The dominant part of compressed data is hidden to the lower frequency sub-band which isn't easy to destroy; then hide the trivial data into the higher frequency sub-band. Finally, when the dominant part of the compressed secret image data is damaged, the error data will be modified by the hamming code method. In addition, when the trivial data of the compressed data of the secret image is damaged, it would affect the data relating to the block only. The proposed method has 2.25 bits/pixels of the hiding capacity. The image quality of stego-image and extracted secret image are measured in terms of peak-signal-to-noise ratio and discusses robustness of noise and compression.
\end{abstract}

Keywords: Image hiding, Data hiding, Stego-image, Secret-image, LSB

\section{Introduction}

With the rapid development of the Internet and multimedia technologies, one can easily conceal a large amount of information in various kinds of digital media. Image hiding embeds a secret image in another image which is usually called a cover image and the hiding procedure is named embedding. After hiding the secret image, the cover image becomes a stego-image $[1,4,6]$. The purpose of image hiding is to embed the secret image from unauthorized people seeking it out.

In recent years, a lot of image hiding technologies [2, 7] have been proposed already. Thien et al. [2] submitted a high hiding-capacity method. It could embed by digit by digit on real-time and reach a higher visual quality than LSB to avoid artificial edges which is caused by LSB. Then, Chan et al. [3] also submitted Optimal Pixel Adjustment Process, OPAP. The concept is according to the method which was submitted by Wang et al. [7]. This method adjusts the last 4 bits of the image to do an optimal pixel process and computes simply. These technologies mostly only focus on the first four requirements, but omit the requirement of robustness. As a result, this paper proposes a BST-based robust image hiding method (BBRIHM) with robustness.

When the dominant compression data of the secret image is damaged, the BBRIHM makes use of the Hamming code technology [8] to detect them and attempts to recover them. If the subordinate compression data of the secret image is changed, the BBRIHM will also try to make only the block related to this data in the secret image be affected.

\section{Data Embedding}

The BBRIHM embeds a secret image $\boldsymbol{I}_{\boldsymbol{s}}$ in a cover image $\boldsymbol{I}_{\boldsymbol{c}}$. Here $\boldsymbol{I}_{\boldsymbol{s}}$ and $\boldsymbol{I}_{\boldsymbol{c}}$ are all gray-level scale images. In order to reduce the data size of $\boldsymbol{I}_{\boldsymbol{s}}$, the BBRIHM adopts BST to encode $\boldsymbol{I}_{\boldsymbol{s}}$ and classifies the compression data in accordance with their importance. After that, it transforms $\boldsymbol{I}_{\boldsymbol{c}}$ into a frequency domain image $\boldsymbol{I}_{\boldsymbol{f}}$ with DWT, and then hides the dominant data of the compression data of $\boldsymbol{I}_{\boldsymbol{s}}$ in lower frequency sub-band of $\boldsymbol{I}_{\boldsymbol{f}}$ and the trivial data in the higher frequency sub-band.

\subsection{Base Switching Transformation}

Generally, the colors of most neighboring pixels in an image are very close. When the pixel colors of a certain image block are all similar, one can use only the minimal pixel color and the differences between the minimal color and all pixel colors in the block to describe the content of this block. Since the color differences among the pixels in the block are all minute, only a few bits are enough to describe each color difference. As a result, it can save a great amount of memory space. We call it base switching transformation method (BST) [9].

The BBRIHM first partitions $I_{s}$ into small blocks of $3 \times 3$ pixels. We named them secret blocks. Suppose $C_{M}$ and $C_{m}$ are the maximal and minimal pixel colors in a certain secret block $B$. It is enough to record the color difference of each pixel in $B$ with only $\left\lceil\log _{2}\left(C_{M}-C_{m}\right)\right\rceil$ bits. In the BST, if the minimal pixel color $C_{m}$ has been changed, all the pixel colors of the whole block will also be altered after decompressing. Therefore, $C_{m}$ is the dominant 
data of the compression data.

\subsection{Hamming Code Appending}

Hamming codes [8] can correct single bit errors or detect two bit errors in a unit data. A unit data is called a code word. The Hamming rule is expressed by the following inequality:

$2^{p} \geq p+c+1$,

where $\boldsymbol{p}$ is the number of parity bits and $\boldsymbol{c}$ is the number of data bits. Such a code is said to be a $(p+c$, c) Hamming code.

Since $\boldsymbol{C}_{\boldsymbol{m}}$ is an eight-bit data, $c=8$. Let $p=4$; $2^{4}(8+4)+1=13$. Let $b_{1} b_{2} \ldots b_{8}$ be the eight bits of $C_{m}$. $\boldsymbol{p}$ is number of parity bits which needs adding to $\boldsymbol{C}_{\boldsymbol{m}}$. It means that four extra parity bits $\boldsymbol{p}_{1} \boldsymbol{p}_{2} \boldsymbol{p}_{3} \boldsymbol{p}_{4}$ are used to detect whether there is something wrong with $\boldsymbol{b}_{1} \boldsymbol{b}_{2} \ldots \boldsymbol{b}_{\boldsymbol{8}}$. We call $\boldsymbol{C}_{\boldsymbol{h}}=\boldsymbol{p}_{1} \boldsymbol{p}_{2} \boldsymbol{p}_{3} \boldsymbol{p}_{4} \boldsymbol{b}_{1} \boldsymbol{b}_{2} \ldots \boldsymbol{b}_{\boldsymbol{8}}$ Hamming code data bits.

Table 1: The corresponding relation of bits

\begin{tabular}{|c|c|c|c|c|c|c|c|c|c|c|c|c|c|c|c|c|}
\hline \multicolumn{17}{|c|}{$\boldsymbol{p}_{1} \boldsymbol{p}_{2} \boldsymbol{p}_{3} \boldsymbol{p}_{4} \boldsymbol{b}_{1} \boldsymbol{b}_{2} \ldots \boldsymbol{b}_{8}$ and $\boldsymbol{e}_{1} \boldsymbol{e}_{2} \boldsymbol{e}_{3} \boldsymbol{e}_{4}$} \\
\hline$-e_{1} e_{2} e$ & 0 & 1 & 2 & 4 & 8 & 3 & 5 & 6 & 9 & & 12 & 7 & & & 14 & 1 \\
\hline uation bit & $\begin{array}{c}\text { No } \\
\text { error }\end{array}$ & & & & & & & & $b_{4}$ & & $\boldsymbol{b}_{6}$ & & $\boldsymbol{b}_{\boldsymbol{8}}$ & - & - & \\
\hline$e_{1}$ & 0 & 1 & 0 & 0 & 0 & 0 & 0 & 0 & 1 & 1 & 1 & 0 & 1 & 1 & 1 & 1 \\
\hline$e_{2}$ & 0 & 0 & 1 & 0 & 0 & 0 & 1 & 1 & 0 & 0 & 1 & 1 & 0 & 1 & 1 & 1 \\
\hline$e_{3}$ & 0 & 0 & 0 & 1 & 0 & 1 & 0 & 1 & 0 & 1 & 0 & 1 & 1 & 0 & 1 & 1 \\
\hline$e_{4}$ & 0 & 0 & 0 & 0 & 1 & 1 & 1 & 0 & 1 & 0 & 0 & 1 & 1 & 1 & 0 & 1 \\
\hline
\end{tabular}

The BBRIHM makes the dominant data bits corresponding to the 4 equations $e_{1}, e_{2}, e_{3}$, and $e_{4}$. Table 1 illustrates their corresponding relations. Equation $e_{i}$ on Table 1 corresponds to the following equation $(i)$, where $\oplus$ is the bitwise EXCLUSIVE OR operation.

$\left\{\begin{array}{l}b_{4} \oplus b_{5} \oplus b_{6} \oplus b_{8} \oplus p_{1}=0 \ldots \ldots \ldots \ldots . \\ b_{2} \oplus b_{3} \oplus b_{6} \oplus b_{7} \oplus p_{2}=0 \ldots \ldots . . . \\ b_{1} \oplus b_{3} \oplus b_{5} \oplus b_{7} \oplus b_{8} \oplus p_{3}=0 . \\ b_{1} \oplus b_{2} \oplus b_{4} \oplus b_{7} \oplus b_{8} \oplus p_{4}=0 .\end{array}\right.$

\subsection{Data Hiding}

When reconstructing $I_{s}$, if the compression data of $B$ has already been destroyed, the BBRIHM will make use of the neighboring blocks of $B$ to estimate the pixel colors of $B$. To guarantee that there are some correct neighboring blocks of $B$ able to estimate the pixel colors of $B$, the BBRIHM classifies all secret blocks of $I_{s}$ into classes 1 and 2 shown in Fig. 2.

\begin{tabular}{|l|l|l|l|}
\hline 1 & 2 & 1 & 2 \\
\hline 2 & 1 & 2 & 1 \\
\hline 1 & 2 & 1 & 2 \\
\hline 2 & 1 & 2 & 1 \\
\hline
\end{tabular}

Fig. 2: Two classes of secret blocks
Discrete wavelet transform (DWT) technique [5] can transform a spatial domain image into a frequency domain image. The DWT coefficients on lower frequency bands are more insensitive to the lossy image processing operations. Therefore, the BBRIHM transforms $I_{c}$ into a frequency domain image $I_{f}$, and hides the dominant and subordinate data of secret blocks in the lower frequency DWT coefficients and the trivial data in the higher frequency DWT coefficients of $I_{f}$.

The BBRIHM hides the dominant data $D_{h}=$ $p_{1} p_{2} p_{3} p_{4} b_{1} b_{2} \ldots b_{8}$ and the subordinate data $S=s_{1} s_{2} s_{3}$ of the compression data of a secrete block $B$ in $L L$ and the trivial data $D_{t}$ in other frequency bands. To prevent the secret image from unauthorized people figuring out any messages about the secret image, the BBRIHM takes a private key $K$ as the seed of a random number generator $G$ which will be used to specify the coefficients veiling the dominant and subordinate data of the compression data of a secret block.

To embed the compression data of a secret block $B$, the BBRIHM employs the random number generator $G$ to randomly selects 15 DWT coefficients $C_{1}^{L L}, C_{2}^{L L}, \ldots, C_{15}^{L L}$ from $L L$ to hide the $D_{h}$ and $S$ of $B$. Here if $B$ is in class 1 , the 4 -th rightmost bits of the 15 coefficients must not have concealed any secret data; otherwise, the 3 -th rightmost bits of the 15 coefficients must not have embedded any secret data. Thereafter, the 15 data bits of $D_{h}$ and $S$ respectively substitute for the 4 -th rightmost bits of the 15 coefficients if $B$ is in class 1 ; otherwise, they replace the 3 -th rightmost bits of the 15 coefficients. Since the 4-th rightmost bits of the 15 coefficients is more insensitive to the lossy image operations than the 3-th rightmost bits, the $D_{h}$ and $S$ of a secret block in class 1 is more robust than those in class 2 in resisting the damage caused by lossy image operation.

Each of the 15 DWT coefficients $C_{1}^{L L}, C_{2}^{L L}, \ldots$, $C_{15}^{L L} \quad$ corresponds to three DWT coefficients respectively in sub-bands $\boldsymbol{L H}, \boldsymbol{H L}$, and $\boldsymbol{H H} . C_{i}^{L L}$ is related to $C_{i}^{L H}, C_{i}^{H L}$, and $C_{i}^{H H}$ which have the same location with $C_{i}^{L L}$ in $\boldsymbol{L} \boldsymbol{L}, \boldsymbol{L H}, \boldsymbol{H L}$, and $\boldsymbol{H H}$ respectively.

Let $\left|D_{t}\right|$ be the size of $D_{t}$. If $\left|D_{t}\right| \leq 45$, the BBRIHM embeds the whole $D_{t}$ in the 45 DWT coefficients; otherwise, it only takes the leftmost 45 bits of $D_{t}$ out to be covered with the 45 DWT coefficients, and abandons the rest of $D_{t}$. The BBRIHM hides the 15 leftmost bits of $D_{t}$ in the 15 corresponding DWT coefficients in $C_{1}^{L H}, C_{2}^{L H}, \ldots$, $C_{15}^{L H}$, the 16-th to 32-th leftmost bits in $C_{1}^{H L}$, $C_{2}^{H L}, \ldots, C_{15}^{H L}$, and the 33-th to 45-th leftmost bits in $C_{1}^{H H}, C_{2}^{H H}, \ldots, C_{15}^{H H}$. If $B$ is in class 1 , the BBRIHM cloaks $D_{t}$ in the second rightmost bits of 
the 45 DWT coefficients; else, in the rightmost bit of the DWT coefficients.

\section{Data Extracting}

To extract the secret image $\boldsymbol{I}_{\boldsymbol{s}}$ from the stego-image, the BBRIHM uses the same private key $\boldsymbol{K}$ as the seed of the random number generator $\boldsymbol{G}$, and transforms the stego-image with spatial format into the image $\boldsymbol{I}_{\boldsymbol{s} t}$ with frequent format by DWT. During extracting the secret block $\boldsymbol{B}$, the BBRIHM finds out $C_{1}^{L L}$, $C_{2}^{L L}, \ldots, C_{15}^{L L}$ from $\boldsymbol{L} \boldsymbol{L}$ via $\boldsymbol{G}$, which ships $\boldsymbol{D}_{\boldsymbol{h}}$ and $\boldsymbol{S}$ of $\boldsymbol{B}$. If $\boldsymbol{B}$ is in class $1, \boldsymbol{D}_{\boldsymbol{h}}$ and $\boldsymbol{S}$ are covered with the 4-th rightmost bits of the 15 coefficients; otherwise, with the 3-th rightmost bits of the 15 coefficients. After searching out $C_{1}^{L L}, C_{2}^{L L}, \ldots, C_{15}^{L L}$, thereupon, $\mathrm{C}_{1}^{\mathrm{LH}}, \mathrm{C}_{2}^{\mathrm{LH}}, \ldots, \mathrm{C}_{15}^{\mathrm{LH}}, \mathrm{C}_{1}^{H L}, \mathrm{C}_{2}^{H L}, \ldots, \mathrm{C}_{15}^{H L}, \mathrm{C}_{1}^{H H}$, $\mathrm{C}_{2}^{H H}, \ldots, \mathrm{C}_{15}^{H H}$ are also decided. If $\left|\boldsymbol{D}_{\boldsymbol{t}}\right| \leq 45, \boldsymbol{D}_{\boldsymbol{t}}$ can be obtained from $\mathrm{C}_{1}^{\mathrm{LH}}, \mathrm{C}_{2}^{\mathrm{LH}}, \ldots, \mathrm{C}_{15}^{\mathrm{LH}}, \mathrm{C}_{1}^{H L}$, $\mathrm{C}_{2}^{H L}, \ldots, \mathrm{C}_{15}^{H L}, \mathrm{C}_{1}^{H H}, \mathrm{C}_{2}^{H H}, \ldots, \mathrm{C}_{15}^{H H}$; else all the binary digits after the 45 -th leftmost bits of $\boldsymbol{D}_{t}$ are given $\mathbf{0}$-bits.

For the BBRIHM, if the compression data of one secret block is changed, only the content of the block may be influenced. $\boldsymbol{D}_{\boldsymbol{m}}$ is the dominant data of the block; suppose it is destroyed, every pixel color in this block is also converted. Therefore, the BBRIHM will try to re-estimate the block if $\boldsymbol{D}_{\boldsymbol{h}}$ which is blemished and may make $\boldsymbol{D}_{\boldsymbol{m}}$ undependable. Provided that one among the equations $\boldsymbol{e}_{1}, \boldsymbol{e}_{2}, \boldsymbol{e}_{3}$, and $\boldsymbol{e}_{4}$ is $\boldsymbol{1}$, some bits in $\boldsymbol{D}_{\boldsymbol{h}}$ have been changed; then the BBRIHM intends to revise $\boldsymbol{D}_{\boldsymbol{m}}$ by side-match method [10].

Let $\boldsymbol{p}_{1} \boldsymbol{p}_{2} \ldots \boldsymbol{p}_{9}$ be the 9 pixels of $\boldsymbol{B}$, and $\boldsymbol{u}_{1} \boldsymbol{u}_{2} \boldsymbol{u}_{3} \boldsymbol{d}_{1} \boldsymbol{d}_{2} \boldsymbol{d}_{3} \boldsymbol{l}_{1} \boldsymbol{l}_{2} \boldsymbol{l}_{3} \boldsymbol{r}_{1} \boldsymbol{r}_{2} \boldsymbol{r}_{3}$ be the neighbors of the top, bottom, left and right pixels of $\boldsymbol{B}$. Fig. 2 shows the neighborhood of $\boldsymbol{B}$. When an error detected via Hamming code, the BBRIHM revises the secret block by side match method. The method estimates $p_{1} p_{2} \ldots p_{9}$ by: $p_{1}=\left(u_{1}+l_{1}\right) / 2 \quad p_{2}=u_{2} \quad p_{3}=\left(u_{3}+r_{1}\right) / 2$ $\begin{array}{lllll}p_{4} & l_{2} & p_{5} & =r_{2} & p_{6}\end{array}=\left(l_{3}+d_{1}\right) / 2 \quad p_{7}=d_{2} \quad p_{8}=\left(r_{3}+d_{3}\right) / 2$ $\boldsymbol{p}_{\boldsymbol{9}}=\sum_{i=1}^{8} p_{i} / 8$.

\begin{tabular}{|l|l|l|l|l|}
\hline & $u_{1}$ & $u_{2}$ & $u_{3}$ & \\
\hline$l_{1}$ & $p_{1}$ & $p_{2}$ & $p_{3}$ & $r_{1}$ \\
\hline$l_{2}$ & $p_{4}$ & $p_{9}$ & $p_{5}$ & $r_{2}$ \\
\hline$l_{3}$ & $p_{6}$ & $p_{7}$ & $p_{8}$ & $r_{3}$ \\
\hline & $d_{1}$ & $d_{2}$ & $d_{3}$ & \\
\hline
\end{tabular}

Fig. 2: The neighborhood of a block in a secret image

\section{Experience}

The purpose of this section is to investigate the performances of the BBRIHM by experiments. These experiments take image Lena as the cover image and image Airplane as the secret image. Fig. 3 demonstrates both images Lena and Airplane respectively with $512 \times 512$ and $384 \times 192$ pixels. PSNR (peak of the signal-to-noise) is often used to measure the similarity between the two images.

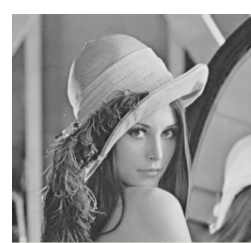

(a) Lena

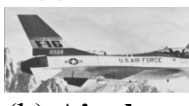

(b) Airplane

Fig. 3: The testing images

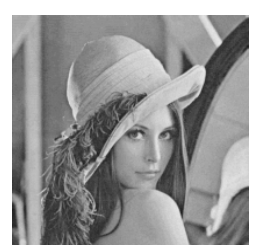

(a) Lena

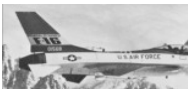

(b) Airplane

Fig. 4: The stego-image and secret images in experiment 1
In the first experiment, Airplane is hidden in Lena by the BBRIHM method. The experimental result demonstrates that the PSNR between the cover image Lena and stego-image is $31.37 \mathrm{~dB}$, and the data hiding rate is 2.25 bits/pixel. After processing Optimal Pixel Adjustment Process [2, 3], PSNR can increase to $33.88 \mathrm{~dB}$ Fig. 4 shows the stego-image and extracted secret image. Table 2 shows PSNRs of stego-image of simple LSB substitution (LSB), Optimal Pixel Adjustment Process (OPAP) [2, 3] and BST-based robust image hiding method (BBRIHM). It can find that the stego-image quality of BBRIHM is between LSB and OPAP.

Table 2: PSNRs of stego-image (dB)

\begin{tabular}{|c|c|c|c|}
\hline & LSB & OPAP & BBRIHM \\
\hline PSNR & 32.08 & 34.44 & 33.88 \\
\hline
\end{tabular}

Deal with the software through PhotoImpact 8 , by putting noises into in stego-images in the second experiment. While dealing with this, the value that we establish the parameter variance respectively is $1 \%, 2 \%$ and $3 \%$. As to the original cover image (Fig. 3 (a)), PSNRs is respectively $33.77,33.36$ and 32.75 $\mathrm{dB}$; and stego-image in Fig. 4 (a), PSNRs is respectively $49.62,42.91$ and $39.16 \mathrm{~dB}$.

Later, extract the hidden data from the stego-image which has already joined noises and rebuild the secret image. This experiment revises the mistake of $D_{\min }$ with $\mathrm{HH}$ and HS methods separately, too. Table 3 shows the image quality (PSNR) of these rebuilt secret images. Results of columns $\mathrm{HH}$ and HS are the PSNR revised with $\mathrm{HH}$ and HS methods.

Table 3: PSNRs of the secret image of reconstruction of the second experiment $(\mathrm{dB})$

\begin{tabular}{|c|c|c|c|c|}
\hline Variance & LSB & OPAP & HH & HS \\
\hline $1 \%$ & 14.68 & 14.62 & 15.53 & 17.53 \\
\hline $2 \%$ & 11.20 & 11.21 & 12.21 & 14.15 \\
\hline $3 \%$ & 9.78 & 9.79 & 10.65 & 12.58 \\
\hline
\end{tabular}


The HS correction method can offer the revision result better than $\mathrm{HH}$ correction method. Because Hamming code can only revise the data that only contains a wrong bit correctly, whether or measure only with wrong bit under two correctly. Table 4 proves the results that revise with $\mathrm{HH}$ correction of $D_{\text {min }}$ of blocks in class 1 and class 2 . It shows that the data was damaged more seriously; revise the $D_{\text {min }}$ of blocks with HH correction method would get the worse revision results. Especially $D_{\min }$ of blocks in class 2 is hidden in the relatively apt damaged place, if we revised the mistake of $D_{\min }$ of blocks in class 2 with $\mathrm{HH}$ correction method, we get this result instead.

Table 4: The result of HH correction method, to stego-image including noisy

\begin{tabular}{|c|c|c|c|}
\hline Variance & $1 \%$ & $2 \%$ & $3 \%$ \\
\hline $\begin{array}{c}\text { No. of error bits in } \boldsymbol{D}_{\boldsymbol{m}} \text { of the blocks } \\
\text { in class 1 without error correction }\end{array}$ & 1310 & 2853 & 4485 \\
\hline $\begin{array}{c}\text { No. of error bits in } \boldsymbol{D}_{\boldsymbol{m}} \text { of the blocks } \\
\text { in class 1 with } \mathbf{H H} \text { correction }\end{array}$ & $\mathbf{9 3 4}$ & $\mathbf{2 5 6 0}$ & $\mathbf{4 4 3 3}$ \\
\hline $\begin{array}{c}\text { No. of error bits in } \boldsymbol{D}_{\boldsymbol{m}} \text { of the blocks } \\
\text { in class 2 without error correction }\end{array}$ & 2680 & 5884 & 9011 \\
\hline $\begin{array}{c}\text { No. of error bits in } \boldsymbol{D}_{\boldsymbol{m}} \text { of the blocks } \\
\text { in class 2 with } \mathbf{H H} \text { correction }\end{array}$ & $\mathbf{2 3 4 3}$ & 5965 & 9314 \\
\hline
\end{tabular}

Deal with JPEG 2000 compression method with PhotoImpact 8 to compress with lossy compression with stego-image. The parameter quality was established as 97, 98, .., 100 respectively. This experiment comes to having wrong $D_{\text {min }}$ to revise with $\mathrm{HH}$ and $\mathrm{HS}$ method separately too. Table 5 demonstrates the result of this experiment. The result in columns $\mathrm{HH}$ and $\mathrm{HS}$ are the rebuilt secret image quality that was revised with $\mathrm{HH}$ and HS method separately. CR is the compression result after JPEG 2000 compression method compressing the stego-image. The following definition is shown:

$$
\mathrm{CR}=\frac{\text { The data size of the original image }}{\text { The data size of compressed image }}
$$

Table 5: Demonstrates the result of this experiment

\begin{tabular}{|c|c|c|c|c|c|}
\hline \multirow{2}{*}{ Quality } & \multirow{2}{*}{ CR } & \multicolumn{4}{|c|}{ PSNR (dB) } \\
\cline { 3 - 6 } & & LSB & OPA & HH & HS \\
\hline 100 & 2.17 & 14.82 & 14.50 & 15.89 & 17.94 \\
\hline 99 & 3.51 & 11.49 & 11.03 & 11.82 & 13.98 \\
\hline 98 & 3.66 & 10.46 & 10.64 & 11.76 & 13.76 \\
\hline 97 & 8.35 & 9.63 & 8.97 & 9.13 & 10.50 \\
\hline
\end{tabular}

\section{Conclusions}

The demand of robustness in image hiding filed is not requested as strongly as it is in watermarking filed. As a result, image hiding method usually neglects the basic demand of robustness. Because of adverse circumstances, when the stego-image is transmitted to the network it would often cause the data transmitted to damage. Also in order to reduce the data transmission time, it is common to compress the image which is going to transmit with lossy compression before transmitting. As a result, this report proposes image hiding method of BBRIHM, this method not only can meet the traditional basic demands of image hiding, but also can resist noise and certain damage in the secret image that is caused by lossy compression. And this method can be offered to 2.25 / the high data hiding rate of pixel.

\section{References}

[1] C. C. Chang, T. S. Chen and L. Z. Chung, "A Steganographic Method Based upon JPEG and Quantization Table Modification," Information Sciences, Vol. 141, No. 1, 2002, pp. 123-138.

[2] C. C. Thien and J. C. Lin, "A Simple and High-Hiding Capacity Method for Hiding Digit-by-Digit data in images based on Modulus function," Pattern Recognition, Vol. 36, No. 13, 2003, pp. 2875-2881.

[3] C. K. Chan, L. M. Cheng, "Hiding data in image by simple LSB substitution", pattern recognition, Vol. 37, No. 3, 2004, pp. 469-474.

[4] J. B. Feng, I. C. Lin, C. S. Tsai, and Y. P. Chu, "Reversible Watermarking: Current Status and Key Issues," International Journal of Network Security, Vol. 2, No. 3, 2006, pp. 161-170.

[5] J. Wang, L. Ji, "A Region and Data Hiding Based Error Concealment Scheme for Images, "IEEE Transformations on Consumer Electronics, Vol. 47, No. 2, 2001, pp. 257-262.

[6] N. F. Johnson, and S. Jajodia, "Steganography: Seeing the Unseen," IEEE Computer, February 1998, pp. 26-34.

[7] R. Z. Wang, C. F. Lin and J. C. Lin, "Image Hiding by Optimal LSB substitution and genetic algorithm," Pattern Recognition, Vol. 34, No. 3, 2001, pp. 671-683.

[8] R. W. Hamming, "Error Detecting and Error Correcting Codes," Bell System Tech. j., Vol. 29, April 1950, pp. 147-160.

[9] T. J. Chuang and J. C. Lin, "New approach to image encryption," Journal of Electronic Imaging, Vol. 7, No. 2, April 1998, pp. 350-356.

[10] T. Kim, "Side Match and Overlap Match Vector Quantizers for Images," IEEE Transactions on Image Processing, Vol. 1, April 1992, pp. 170-185. 